\title{
Cambio de la estructura productiva en Chile, 1986-1996: producción e interdependencia industrial
}

\author{
José Miguel Albala-Bertrand
}

$\mathrm{E}$

n estudios anteriores, el autor mostró que el modelo de desarrollo adoptado por Chile difería significativamente del aplicado por los países exitosos del sudeste asiático. Hasta 1986, la base productiva de la economía chilena era relativamente débil y de poca complejidad tecnológica, y la penetración de insumos importados se hacía principalmente a expensas de la escasa intermediación productiva interna. Ni la industrialización manufacturera ni la interdependencia industrial resultantes parecían favorecer la exportación de manufacturas capaces de sustentar un desarrollo industrial dinámico basado en los mercados externos. El presente artículo analiza los cambios en la estructura productiva chilena entre 1986 y 1996, y en general hasta el 2000, utilizando una metodología similar a la de los estudios previos. Concluye que, pese a las notables tasas de crecimiento del período analizado, la economía chilena aún parece ser relativamente débil como base para acrecentar de manera sostenible un desarrollo económico y Departamento de Economía, Queen Mary, tecnológico más complejo. 


\section{I}

\section{Introducción}

El presente artículo continúa trabajos anteriores del autor sobre los cambios en la estructura productiva y en la interdependencia industrial registrados en Chile entre 1960 y 1990 (Albala-Bertrand, 1999a y 1999b). Los resultados de esos trabajos se compararon luego con los obtenidos en etapas de desarrollo equiparables por algunos países de industrialización reciente, con el propósito de evaluar en qué medida las políticas basadas en el Consenso de Washington producirían estructuras económicas en general tan satisfactorias como las de los países que habían aplicado el modelo japonés/ asiático.

Dicho brevemente, en las últimas décadas han surgido en competencia dos grandes modelos de políticas de desarrollo orientadas al mercado: el modelo japonés/asiático y el modelo del Consenso de Washington. Ambos destacan la importancia de la estabilidad macroeconómica y del desarrollo liderado por las exportaciones y la iniciativa privada. Sin embargo, difieren fundamentalmente en su visión del papel que desempeñan el mercado y el Estado en el desarrollo y, por ende, de la gama y tipo de políticas económicas disponibles (Wade, 1990; Stiglitz, 1996; Chang y Grabel, 2004).

El modelo del Consenso de Washington apunta principalmente a asegurar la liberalización del comercio mediante la eliminación de todas o casi todas las barreras que lo limitan, y a lograr la estabilidad macroeconómica tanto mediante el equilibrio presupuestario y las privatizaciones como a través de la desregulación y la liberalización para permitir que las señales de precios operen eficazmente en la economía (Williamson, 1990). El gobierno ha de reducirse a un nivel mínimo y tener carácter subsidiario. Las políticas económicas, por lo tanto, deben ser neutrales y operar automáticamente a través del mercado. La versión extrema de este modelo postula que las fallas del mercado son siempre menos costosas que la intervención y las fallas del gobierno, de manera que en la mayoría de los casos, por no decir siempre, el papel del gobierno es irrelevante (Krueger, 1990).

Por su lado, el modelo japonés/asiático reconoce expresamente la importancia de la estabilidad macroeconómica y de la orientación exportadora, pero desconfía en cierta medida de los mecanismos del "libre" mercado. El Estado ha de ser un pivote esencial para establecer la dirección y el ritmo del desarrollo, sobre todo, aunque no exclusivamente, en lo que toca a la política industrial. Esto significa que para lograr un desarrollo dinámico (en vez de estático) y estratégico (en vez de ciego) es indispensable contar con políticas selectivas (no neutrales) y reguladoras (no automáticas). El gobierno, entre otras funciones, ha de contribuir a la creación de ventajas comparativas dinámicas mediante un proteccionismo informado en beneficio de líneas de exportación potenciales, y a la coordinación de la economía en su conjunto. ${ }^{1}$ Por lo tanto, incluso si no participara directamente en las actividades productivas, haría un aporte esencial a la tarea de orientar y agilizar los cambios en la estructura productiva.

Para comparar ambos modelos, en los trabajos anteriores del autor se eligieron como objeto de análisis las economías en las cuales ellos se habían aplicado con mayor éxito: Chile en el caso del Consenso de Washington, y la economía taiwanesa y la República de Corea en el caso del modelo japonés/asiático. Esos estudios mostraron que en Chile, contrariamente a lo sucedido en los países del sudeste asiático y cualesquiera hayan sido los otros logros de la experiencia chilena, tanto la interdependencia industrial como la industrialización basada en las exportaciones de manufacturas seguían siendo bases endógenas débiles y poco promisorias para sustentar un desarrollo industrial dinámico liderado por los mercados externos (AlbalaBertrand, 1999a y 1999b). Todavía en 1990, transcurridos al menos 15 años desde que Chile comenzó esta experiencia y aun teniendo en cuenta las dispares situaciones iniciales, la economía y la estructura productiva chilenas diferían significativamente de aquellas taiwanesas y sudcoreanas.

Este artículo busca averiguar si hubo alguna convergencia entre las respectivas situaciones en el período 1986-1996, que en términos de crecimiento parece haber sido el más exitoso de la historia económica chilena, con tasas medias anuales de 7\%. Sin embargo, a

\footnotetext{
${ }^{1}$ Véase Wade (1990); Chang (1996); Stiglitz (1996 y 1998); Stiglitz y Uy (1996); Lall (1996 y 1997); Islam y Choudhury (2000), y
} Chang y Grabel (2004). 
partir de 1986 Chile no parece haber aplicado una política industrial, y el gobierno generalmente ha favorecido las políticas del Consenso de Washington. Esto no quiere decir que el gobierno no haya contribuido significativamente a la elaboración de conjuntos de políticas o a la corrección de otras para sacar mejor provecho de la estrategia seguida. Tampoco significa que no haya existido una política pública dirigida a dar sustento a algunas industrias manufactureras básicas. ${ }^{2}$ En cambio, sí significa que tales estrategias no fueron sistemáticas ni generales y que no favorecieron el tipo de política industrial que se asocia al desempeño exitoso de las economías sudcoreana y taiwanesa, es decir, al modelo japonés/asiático. Con el propósito señalado, este estudio analiza los datos de insumo-producto correspondientes al período 19861996, que en general representan alguna actualización hacia el año $2000 .^{3}$

Para evitar equívocos, conviene aclarar cuál es el tipo de cambio estructural que puede captarse aplicando la metodología que se describe en la sección siguiente. El cambio de la estructura productiva, que es

\section{II}

\section{Metodología}

El primero en presentar una metodología para evaluar el cambio en la estructura de la producción bruta fue Chenery (1960). Esa metodología se basaba en las tablas de insumo-producto y fue posteriormente perfeccionada por varios autores. ${ }^{5}$ En la práctica, el método de descomposición utilizado en este trabajo se asemeja más a los de Wyckoff y Sakurai (1992) y de AlbalaBertrand (1999a). Este método para analizar el cambio en la estructura productiva aprovecha la mayoría de las ventajas del enfoque de insumo-producto $\mathrm{y}$, al mismo tiempo, evita muchas de sus limitaciones: es decir, hace que pierdan importancia sus inconvenientes habituales para realizar proyecciones (Bulmer-Thomas, 1982; Ciaschini, 1988).

Una de las ventajas, que aquí aprovechamos cabalmente, es la posibilidad de descomponer el cambio estructural global de un período determinado en las

\footnotetext{
2 Véase Achurra (1995); Agosin (1997); Moguillansky (1999); Ffrench-Davis y Stalling (2001)

${ }^{3}$ Para mayores detalles, véase Albala-Bertrand (1999a y 1999b).
}

el tema central de este artículo, puede surgir de modificaciones institucionales o de diferencias de crecimiento entre los distintos segmentos de una determinada estructura institucional. Entre 1975 y 1985 Chile experimentó grandes transformaciones institucionales, y entre 1986 y 1996 se agregaron cambios institucionales significativos, aunque con mayor equilibrio y moderación que en los diez años anteriores. Las variaciones resultantes en la composición de la producción bruta que muestra este artículo se refieren al período 1986-1996, en el cual se conservó en líneas generales la estructura institucional del período anterior. Por lo tanto, es probable que esas variaciones tengan un importante componente institucional, vinculado tanto con la estrategia global aplicada a partir de 1975 como con las modificaciones de política efectuadas en 1986-1996. ${ }^{4}$ Así, el método que se ha utilizado capta algunos cambios sectoriales e intersectoriales que se produjeron en la economía chilena durante este último período, en el marco prevaleciente de instituciones y estrategias de política que podrían haber afectado a variables económicas como el crecimiento y la productividad. fuentes de demanda que contribuyeron a él, tanto respecto del complejo industrial en su conjunto como de cada una de las industrias que lo componen. Las fuentes de demanda, sin embargo, no explican el cambio estructural en términos de causalidad, sino solamente en términos de concomitancia ex post. Por lo tanto, constituyen bases empíricas útiles para efectuar un análisis de política estructuralmente amplio, el cual excedería los alcances de este trabajo.

A su vez, la metodología usada para evaluar los cambios estructurales en la interdependencia industrial —entendida como el cambio estructural en la interacción interna de las industrias a nivel de bienes intermedios - se basa en un análisis de multiplicador aplicado a los eslabonamientos hacia adelante y hacia atrás. La principal diferencia entre el análisis que se hace aquí y el análisis estándar de los eslabonamientos es que,

\footnotetext{
${ }^{4}$ Véase Albala-Bertrand (1996).

${ }^{5}$ Véase, por ejemplo, Dervis, de Melo y Robinson (1982); Kubo, de Melo y otros (1986); Sakurai (1990).
} 
conforme a la teoría de insumo-producto, el análisis estándar aplica un criterio ex ante para abordar el potencial de planificación de la expansión económica (Hirschman, 1958 y 1977; Syrquin, 1992). En cambio, como nuestro objetivo es examinar la alteración efectiva de los eslabonamientos estructurales a lo largo del tiempo, en nuestro análisis los eslabonamientos representan la interdependencia real o efectiva (o ex post) de la economía en los años 1986 y 1996. Con esto también se reduce en gran medida lo restrictivo de los supuestos necesarios para el análisis estándar. ${ }^{6}$

Por lo tanto, a partir del marco general del enfoque de insumo-producto podemos construir algunas ecuaciones que nos permitan analizar tanto el cambio de la estructura productiva como el de la interdependencia industrial. ${ }^{7}$ Así, en la matriz representamos el cambio estructural, en primer lugar, por una ecuación para las variaciones de la producción bruta:

$$
\begin{aligned}
\Delta X & =B_{0} \hat{U}_{o}{ }^{F} \Delta F+B_{0} \Delta E+B_{0} \Delta \hat{U}^{F} F_{1} \\
& +B_{0} \Delta \hat{U}^{W} W_{1} i+B_{0} \hat{U}_{0}{ }^{W} \Delta A X_{1}
\end{aligned}
$$

donde $\Delta X$ es la variación de la producción bruta, $B$ una versión adaptada de la matriz inversa de Leontief, $U$ los bienes intermedios nacionales como proporción del total de bienes intermedios (superíndice $W$ ) y la demanda final interna como proporción de la demanda final total (superíndice $F$ ), $F$ es la demanda final, $E$ la demanda externa y $W$ la demanda de bienes intermedios. Los subíndices 0 y 1 corresponden, respectivamente, al año inicial y final (es decir, 1986 y 1996). La descomposición de la tasa de crecimiento de la producción bruta puede obtenerse dividiendo la ecuación [1] por $X_{0}$. En términos de variación o crecimiento, cada uno de los cinco términos en el lado derecho de la ecuación [1] representa una contribución directa e indirecta a la demanda total de la producción bruta de la economía y significa lo siguiente:

$$
\begin{aligned}
B_{0} \hat{U}_{0}^{F} \Delta F= & \text { contribución del incremento de la de- } \\
& \text { manda interna final (FDE) } \\
B_{0} \Delta E= & \text { contribución del incremento de la de- } \\
& \text { manda de exportaciones (EDE); }
\end{aligned}
$$

\footnotetext{
${ }^{6}$ Véase también Albala-Bertrand (1999b).

${ }^{7}$ Véase una versión completa de estos modelos en Albala-Bertrand (1999a y 1999b).
}

$$
\begin{aligned}
& B_{0} \Delta \hat{U}^{F} F_{1}=\text { contribución de la sustitución de impor- } \\
& \text { taciones de bienes finales (ISF) }{ }^{8} \\
& B_{0} \Delta \hat{U}^{W} W_{1} i=\text { contribución de la sustitución de impor- } \\
& \text { taciones de bienes intermedios (ISW); } \\
& B_{0} \hat{U}_{0}^{W} \Delta A X_{1}=\text { contribución de los cambios en los co- } \\
& \text { eficientes de insumo-producto (IOC) di- } \\
& \text { rectos. }{ }^{9}
\end{aligned}
$$

Enseguida, para calcular las variaciones en la participación de la producción bruta utilizamos la siguiente ecuación:

$$
\begin{aligned}
\delta X & =B_{0} \hat{U}_{o}{ }^{F} \delta F+B_{0} \delta E+B_{0} \Delta \hat{U}^{F} F_{1} \\
& +B_{0} \Delta \hat{U}^{W} W_{1} i+B_{0} \hat{U}_{0}{ }^{W} \Delta A X_{1}
\end{aligned}
$$

donde todos los términos tienen el mismo significado que en la ecuación anterior, pero $\delta$ representa la desviación respecto del crecimiento equilibrado (es decir, el que habría predominado si todas las actividades productivas hubieran crecido a la misma tasa media que la economía). Por lo tanto, esto es indicación de cambio estructural mediante variaciones de la participación de dichas actividades durante el período. Cabe destacar que los tres últimos términos del lado derecho de la ecuación son los mismos que antes. El significado es análogo al de la ecuación [1], pero se refiere al valor absoluto del cambio en la participación $(\delta X)$ y a la variación relativa de la participación $\left(\delta X / X_{t 1}\right)$, más que al crecimiento absoluto $(\Delta X)$ y a la tasa de crecimiento $\left(X^{1} \Delta X\right)$.

Para analizar la interdependencia industrial, recurrimos al cálculo de los eslabonamientos hacia atrás y hacia adelante. Definimos el eslabonamiento hacia

\footnotetext{
${ }^{8}$ Los términos 3 y 4 son positivos cuando ha habido un incremento de la sustitución de importaciones en el período. A la inversa, una caída en la sustitución de importaciones significa un aumento de la entrada de importaciones.

${ }^{9}$ Esto representa la variación de la demanda durante el período, exclusivamente como resultado de la variación en los coeficientes de insumo-producto del sistema, ponderados por el valor medio de $\mathrm{X}_{1}$. El hecho de que en una industria determinada este término sea positivo quiere decir que la proporción de la producción de esta industria que es utilizada como insumo para la producción de otras industrias es mayor que en el año base. En otras palabras, hay un incremento de la contribución intermedia directa de esa industria a la economía. Esto representa una forma de eslabonamiento directo hacia atrás (demanda) del resto de la economía con esa industria, o bien una forma de eslabonamiento directo hacia adelante (oferta) de esa industria con el resto de la economía durante el período (Hirschman, 1977).
} 
atrás $(B L)$ como la dependencia directa e indirecta de una industria determinada respecto de todas las industrias, puesto que estas le suministran insumos intermedios para su propia producción. Se espera de las industrias dinámicas que aumenten sus eslabonamientos hacia atrás y se especialicen a medida que se desarrollen, sea porque necesitan más cantidad de una combinación más variada de insumos o porque dejaron de producir ellas mismas parte de esos insumos. En consecuencia, se espera que las industrias de vanguardia, que tienen una fuerte integración vertical, den impulso a la economía y, si son tecnológicamente avanzadas, que mejoren el nivel tecnológico de la economía en general. En la matriz, los eslabonamientos hacia atrás $(B L)$ se expresan de la siguiente manera:

$$
\begin{gathered}
B L=i^{\prime} C^{-1}(\text { eslabonamiento hacia atrás, } \\
\text { vector de fila) }
\end{gathered}
$$

en que $C^{-1}$ representa una matriz inversa de Leontief. Teóricamente, se espera que las industrias líderes dinámicas exhiban eslabonamientos superiores al promedio y una distribución más equitativa que en otras industrias.

Finalmente, para apreciar la variación en el tiempo de los valores de los eslabonamientos hacia atrás $B L$, simplemente calculamos la primera diferencia de este valor entre dos años determinados. Sin embargo, como la matriz $C$ incluye bienes intermedios tanto nacionales como importados, es necesario descomponer para distinguir entre ambos. Así:

$$
\begin{gathered}
\Delta B L=i^{\prime} \Delta C^{-1}=i^{\prime}\left(C_{0}^{-1} \Delta A^{d} C_{1}^{-1}\right) \\
+i^{\prime}\left(C_{0}^{-1} \Delta A^{m} C_{1}^{-1}\right) \quad(\text { descomposición de } \Delta B L)
\end{gathered}
$$

en que $A$ es una matriz estándar de los coeficientes de los insumos. Los superíndices $d$ y $m$ corresponden a "nacional" e "importado", respectivamente. La ecuación [4] es vector de fila de las diferencias de los eslabonamientos regresivos entre dos años, " 0 " y " 1 ", clasificados en contribuciones nacionales e importadas. Esto puede expresarse de manera más útil en función de las tasas de crecimiento (o tasas de variación), dividiendo la ecuación [4] por el valor de $B L$ en el año inicial, como aparece más adelante en el cuadro 1.

Igual que en el caso de los eslabonamientos hacia atrás, definimos los eslabonamientos hacia adelante $(F L)$ como la dependencia directa e indirecta de todas las industrias respecto de una industria determinada, porque necesitan los insumos intermedios que ella proporciona para ocuparlos en su propia producción. Como los valores de $B L$ y $F L$ no tienen por qué ser similares, para formarnos una impresión más cabal de las transacciones totales de bienes intermedios debemos calcular el valor de $F L$. El procedimiento utilizado para hacerlo es el mismo que usamos para calcular el valor de $B L$. Luego, en la matriz los eslabonamientos hacia adelante $(F L)$ se expresan de la siguiente manera:

$$
\begin{aligned}
F L=C^{*-1} i & (\text { vector de columna de los } \\
& \text { eslabonamientos hacia adelante) }
\end{aligned}
$$

donde $C^{*-1}$ representa una matriz inversa de Leontief de los coeficientes de la oferta (en vez de la demanda) directa e indirecta de insumos. Una vez más, se supone que en las industrias más importantes los eslabonamientos superiores al promedio se distribuirán más equitativamente que en las demás industrias.

Finalmente, la descomposición en primera diferencia de la ecuación [5] nos da el siguiente valor de $\triangle F L$ :

$$
\begin{aligned}
\Delta F L= & \left(\Delta C^{*-1}\right) i=\left(C^{*-1} \Delta A^{* d} C_{1}^{*-1}\right) i \\
& +\left(C_{1}^{*-1} \Delta A^{* m} C_{1}^{*-1}\right) i
\end{aligned}
$$

donde $A^{*}$ es una matriz de los coeficientes de la oferta directa de insumos y los superíndices $d$ y $m$ corresponden a "nacional" e "importado", respectivamente. Esta ecuación es un vector de columna de las diferencias de los eslabonamientos hacia adelante entre los años 0 y 1 , desagregadas en contribuciones nacionales e importadas. Al igual que en el caso anterior, esto también puede expresarse de manera más útil en función de las tasas de crecimiento (o tasas de variación), dividiendo la ecuación [6] por el valor de $F L$ en el año inicial. 


\section{III}

\section{Análisis de los resultados}

Para estos efectos, se utilizó aquí una desagregación de 28 actividades productivas y algunos subgrupos importantes. El estudio se centra en el período 1986-1996, y lo compara en líneas generales con un período de referencia, el de 1977-1986, que fuera analizado anteriormente en Albala-Bertrand (1999a y 1999b). Este período de referencia se utiliza solamente para ilustrar las tendencias generales, ya que en los estudios mencionados los cálculos se hicieron a partir de los precios a los usuarios y no de los precios básicos, que no figuraban en las tablas de insumo-producto originales de 1977. ${ }^{10} \mathrm{El}$ análisis considera dos aspectos principales: el cambio estructural en la producción bruta y el cambio estructural en la interdependencia industrial.

\section{Cambio estructural en la producción bruta}

El cuadro 1 muestra el cambio tanto de la participación de las industrias o servicios en la producción bruta como de las tasas de crecimiento de esas actividades, en el período 1986-1996. Comenzaremos por examinar los tres grandes sectores productivos, a saber, el sector primario o de recursos naturales, el sector secundario o manufacturero (que en el cuadro incluye la construcción) y el sector terciario o de servicios.

\section{a) Análisis agregado}

A nivel de los tres grandes sectores - primario, secundario y terciario-, el grado de cambio estructural de la producción bruta durante el período 19861996 al parecer fue similar al grado de cambio observado en el período anterior, pero las tasas de crecimiento de la producción bruta fueron significativamente más altas. La caída de la participación del sector primario, que pareció haberse detenido en 1977-1986, se reanudó en 1986-1996 y sumó otros 3,1 puntos porcentuales. En consecuencia, en 1996 el sector primario representaba el 16,3\% de la producción bruta total. En cuan-

\footnotetext{
${ }^{10}$ Utilizamos los cuadros de insumo-producto de 1986 y 1996 . Este último es el más reciente disponible sobre Chile, se publicó en 2001 y apareció en la Internet en 2002. Cuando fue posible, esos cuadros fueron deflactados a nivel de sectores, distinguiendo bienes intermedios, valor agregado, producto final y producto bruto total a precios constantes de 1986. Para estos efectos utilizamos los deflactores implícitos de las cuentas nacionales.
}

to al sector secundario, en 1986-1996 este contrarrestó en parte el apreciable aumento de su participación logrado en el período anterior, pues descendió 2,8 puntos porcentuales, con lo cual en 1996 representó $35,9 \%$ de la producción bruta total. En el sector terciario parece haber sucedido lo contrario, al revertirse la caída que había experimentado en el período 19771986 y aumentar su participación en 5,9 puntos porcentuales, llegando a representar un $47,8 \%$ de la producción bruta total en 1996. A lo largo del período estudiado las tasas de crecimiento fueron excepcionalmente altas en los tres sectores, y sobre todo en el terciario, que en el período anterior parecía ir muy a la zaga de los otros dos.

Para la economía en su conjunto, el único componente de la demanda que al parecer contribuyó positivamente a las variaciones en la participación de los distintos sectores en 1986-1996 fue una significativa mejora de la intermediación directa, representada por los coeficientes de insumo-producto (IOC). Esto significa que la demanda de insumos para la producción de todos los sectores, especialmente el secundario y el terciario, contribuyó a elevar las tasas de crecimiento y la participación de estos sectores dentro de la economía. El aporte de todos los demás factores a la participación en la producción fue negativa, en especial el incremento de la demanda final (FDE) y la sustitución de importaciones de bienes finales (ISF) y de bienes intermedios (ISW). Incluso el incremento de la demanda de exportaciones, que en el período 1977-1986 parece haber hecho el aporte mayor, exhibió un signo negativo en 1986-1996. No obstante, tanto la demanda interna como las exportaciones y, en menor medida la intermediación, parecen haber sido los factores que más contribuyeron a la elevada tasa media de crecimiento (véase el cuadro 1). La impresión es que esta tasa solo se vio afectada por la sustitución de importaciones, pero en pequeña medida. Sin embargo, los resultados globales ocultan el carácter heterogéneo del cambio estructural, por lo cual a continuación pasaremos a efectuar un análisis desagregado.

\section{b) Análisis desagregado}

A este nivel de desagregación examinaremos actividades productivas incluidas en los sectores de recursos naturales, de manufactura o de servicios. 

1986 y 1996, y tasas de crecimiento, por actividades productivas y por sectores ${ }^{a}$ (Precios básicos constantes en pesos de 1986)

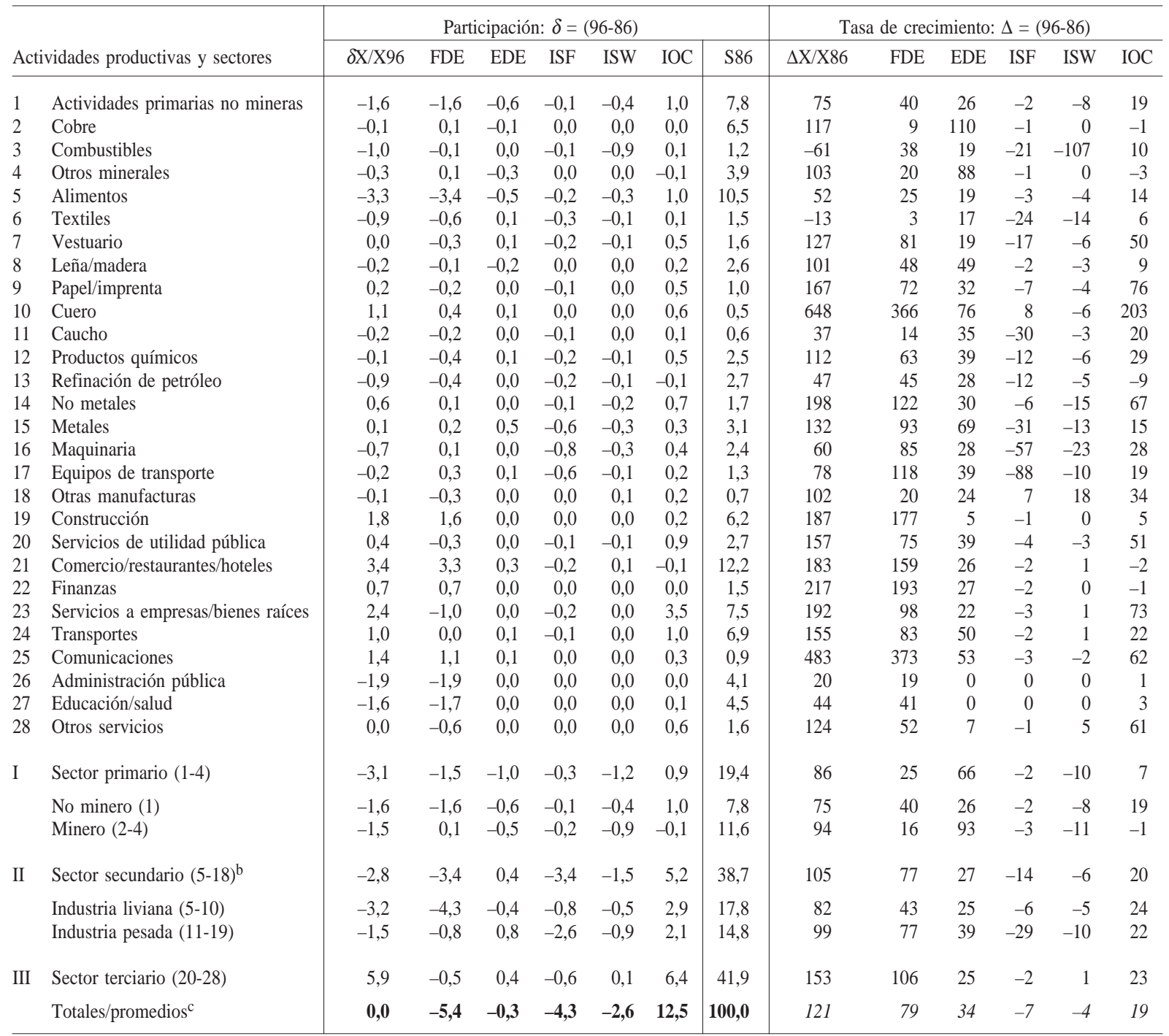

Fuente: Elaboración propia sobre la base de Banco Central de Chile (1993 y 2001a).

a $\mathrm{BP}:$ Precios básicos.

$\delta \mathrm{X} / \mathrm{X}$ : Variación de la participación en la producción bruta $(=\Delta \mathrm{S})$ (puntos porcentuales).

$\Delta \mathrm{X} / \mathrm{X}$ : Tasa de crecimiento de la producción bruta (porcentaje).

FDE : Incremento de la demanda final (puntos porcentuales).

EDE : Incremento de la demanda de exportaciones (puntos porcentuales).

ISF : Sustitución de importaciones de bienes finales (puntos porcentuales).

ISW : Sustitución de importaciones de bienes intermedios (puntos porcentuales).

IOC : Variación de los coeficientes de insumo-producto (puntos porcentuales).

S86 : Participación en la producción bruta en 1986 (porcentaje).

b El sector secundario (manufacturero) incluye la construcción.

c Las cifras en negrita corresponden a totales; las cifras en cursiva corresponden a promedios ponderados. 


\section{i) El sector primario o de recursos naturales (cua-} dro 1, ramas 1 a 4). En el período 1986-1996, a diferencia de lo ocurrido en el período anterior, se redujo la participación en la producción bruta de todas las industrias de este sector, en especial las no mineras, aunque la participación de la industria del cobre continuó siendo prácticamente igual. Todas las ramas, especialmente la cuprífera, exhibieron aumentos significativos de las tasas de crecimiento de su producción bruta; la excepción fueron los combustibles, con una caída de $61 \%$. Sin embargo, todas las industrias del sector primario crecieron a tasas inferiores al promedio de la economía, lo cual explica la caída de su participación. Sus tasas de crecimiento aún significativas parecen deberse principalmente al incremento de la demanda de productos finales (FDE) de la actividad no minera y al aumento de la demanda de exportaciones (EDE) de la actividad minera. La disminución señalada en la rama de combustibles parece haber obedecido principalmente a grandes bajas en la sustitución de importaciones, sobre todo de bienes intermedios (ISw). Como era de esperar, las altas tasas de crecimiento del cobre y otros minerales se debieron principalmente a la demanda de exportaciones, mientras que el aporte de la intermediación parece haber sido casi nula, lo que significa que posiblemente se acentuó el carácter de industria enclave que ha tenido la minería en Chile. En 1986-1996, contrariamente a lo sucedido en el período anterior, la intermediación parece haber contribuido bastante al crecimiento de la producción de las industrias no mineras del sector primario, lo que puede significar que esas industrias se han integrado más a la economía en general.

En síntesis, diremos en primer lugar que la industria del cobre pareció conservar una participación en la producción bruta similar a la de 1986, pero con una tasa de crecimiento significativamente más elevada, lo que se debería al apreciable respaldo de medidas de política durante el período. En 1996, la participación de esta industria en la producción total superó el $6 \%$, lo que favoreció tanto el crecimiento en general como la generación de divisas. Segundo, las industrias primarias no mineras prácticamente duplicaron en 19861996 la tasa media de crecimiento del período anterior. La demanda interna y las exportaciones, así como la intermediación, contribuyeron de manera importante a esta recuperación. Tercero, al parecer la intermediación mejoró en la rama primaria no minera y por ende en la de combustibles, pero empeoró algo en el caso del cobre y de otros minerales. Al parecer la agricultura y las agroindustrias estuvieron más integradas en el con- junto de la economía, mientras que las industrias extractivas iban un poco en sentido contrario o quedaban a la zaga.

Medidas en función del producto interno bruto (PIB), la participación de la minería aumentó entre 1996 y el 2000, mientras que la de la industria primaria no minera (excepto la pesca) se redujo significativamente, con lo cual la participación del sector primario en el año 2000 fue casi igual que en 1996 (Banco Central de Chile, varios años). Esto indica que, en general, el sector primario ha crecido a un ritmo semejante al de la economía en su conjunto, pero con algunos cambios estructurales en su interior. El PIB se refiere solo al componente de demanda final de la producción bruta, pero, a diferencia de esta, se calcula a precios de mercado y no a precios básicos, esto es, incluye costos de transporte, márgenes de utilidad de los mayoristas e impuestos indirectos netos. En consecuencia, aunque el ritmo de crecimiento y la estructura del PIB pueden dar una indicación general sobre el comportamiento de la producción bruta en el mismo período, la confirmación solo se tendrá cuando el Banco Central dé a conocer tablas actualizadas de insumo-producto.

ii) El sector secundario o manufacturero (cuadro 1, ramas 5 a 19). En el período de referencia (19771986) al parecer aumentó la participación del sector manufacturero en la producción bruta total, pero solo gracias a la industria liviana, ya que se redujo la participación de la industria pesada, que constituye la parte tecnológicamente más avanzada del sector. En cambio, durante el período en estudio (1986-1996) disminuyó la participación de ambas: la de la industria liviana en $3,2 \%$ y la de la industria pesada en 1,5\%. La caída de la industria liviana parece haberse debido principalmente al desempeño de la industria textil, cuyo crecimiento fue negativo, y de la industria alimentaria, cuyo crecimiento medio fue bastante inferior al del sector y al de la economía en su conjunto. Las tasas de crecimiento de la industria pesada fueron altas y positivas, pero generalmente inferiores al promedio de la economía. Las ramas que más perdieron participación en la producción bruta fueron la refinación de petróleo y la maquinaria, pero también los productos químicos, los equipos de transporte y otras manufacturas. En cambio, las industrias de metales y no metales exhibieron tasas de crecimiento superiores al promedio y, así, un leve incremento de su participación en la producción total. La construcción conservó su tendencia alcista, con una tasa de crecimiento bastante superior al promedio en el período, y aumentó su participación en 1,8 puntos porcentuales. 
Entre los factores que ayudaron a elevar las tasas de crecimiento estuvieron la demanda interna y las exportaciones, pero también la intermediación directa, especialmente en el caso de prendas de vestir, papel/imprenta, industrias no metálicas y maquinaria. Por su parte, un factor adverso al crecimiento fue la sustitución de importaciones ${ }^{11}$ de bienes tanto finales como intermedios, en especial en las industrias de caucho, metales, maquinaria y equipos de transporte. Al parecer había menos capacidad interna de producir esos bienes y, por lo tanto, la penetración de las importaciones fue mayor. En cuanto a los cambios de la participación en la producción, el hecho de que la tasa de crecimiento de la mayoría de las industrias del sector haya sido inferior al promedio se tradujo en una baja de sus participaciones en el período. Contribuyeron a este resultado todos los factores, salvo la intermediación (IOC) y muy secundariamente el incremento de las exportaciones (EDE). Cabe destacar que, salvo en el caso de las industrias elaboradoras de metales, el aporte del aumento de las exportaciones a la participación del sector secundario en la producción se estancó o tuvo signo negativo. Esto significa, simplemente, que incluso de ser positiva, la contribución de las exportaciones a las tasas de crecimiento estuvo bajo el promedio de la economía. Por lo que toca a la construcción, que en 1996 generó casi 8\% de la producción bruta total, su participación aumentó en forma sostenida; como era de prever, este incremento obedeció principalmente a la expansión de la demanda interna, pero en cierta medida también a la intermediación directa.

En síntesis, señalaremos en primer lugar que en el período analizado la participación de la industria pesada en la producción registró una baja relativa, no absoluta, especialmente en industrias como refinación de petróleo, maquinaria, equipos de transporte y otras manufacturas; pero a la vez mostró un leve incremento en las industrias de metales y de no metales. Segundo, aunque la caída señalada se atribuye sobre todo al aumento general de las importaciones, la intermediación directa también hizo un aporte muy importante que impidió que la caída fuera mayor. El alza de las exportaciones también realizó una pequeña contribución. Tercero, las industrias livianas vieron caer su participación en la producción, pese a sus altas tasas de crecimiento (salvo en la industria textil). Cuarto, esta caída se relacionó principalmente con la entrada de importaciones y con el hecho de que tanto la de-

\footnotetext{
${ }^{11}$ Respecto a la forma en que se calculó aquí el aporte de la sus-
} titución de informaciones, véase la nota 8. manda interna como el aumento de las exportaciones fueran inferiores al promedio de la economía. Quinto, hubo también una contribución significativa de la intermediación directa, lo que podría indicar que las industrias del sector secundario se integraron más que antes al resto de la economía, pero que probablemente se debió sobre todo a la entrada de importaciones, como se verá más adelante. Sexto, en términos relativos parece haber habido una desindustrialización generalizada tanto en la industria liviana como en la pesada. La participación de la industria liviana, que había mostrado una tendencia positiva en el período de referencia (1977-1986), al parecer se revirtió en gran medida en el período analizado (1986-1996). Finalmente, la participación en la producción bruta de la rama de la construcción mantuvo una tendencia al alza gracias a la expansión de la demanda interna.

Medida en función del PIB, la participación del sector manufacturero en la producción bruta experimentó una baja significativa, de alrededor de $4 \%$, y en el año 2000 perdió otro punto porcentual. Esto se debió a que las tasas de crecimiento de las industrias de este sector fueron inferiores al promedio de la economía, aunque no negativas, salvo en el caso de la industria textil. También hay algunos indicios de que dicha caída relativa no estuvo acompañada de aumentos de la productividad (Mujica, 1989; Ominami, 1991; Pizarro, Raczynski y Vial, 1996). Esto podría ser preocupante para una economía que pretenda alcanzar un desarrollo sostenible liderado por las exportaciones (Ffrench-Davis y Sáez, 1995; Albala-Bertrand, 1999a).

iii) El sector terciario o de servicios (cuadro 1, ramas 20 a 28). Contrariamente a lo sucedido en el período 1977-1986, en 1986-1996 la mayoría de las industrias de este sector aumentaron de manera significativa su participación en la producción bruta. Entre las principales actividades que generaron este incremento se cuentan: comercio, restaurantes y hoteles; servicios a empresas/bienes raíces; transporte, y comunicaciones. Es lo que cabía esperar en una economía que ha tenido un alto crecimiento del PIB en un determinado período. Sin embargo, no fue la expansión de la demanda interna lo que más influyó en este resultado, sino una mucho mayor participación de la intermediación directa. Por lo demás, algunas importaciones de bienes finales se contrapusieron a este desempeño positivo. Con todo, hay algunos motivos de preocupación, ya que tanto en administración pública como en educación/salud las tasas de crecimiento fueron muy inferiores al promedio de la economía, y la participación en el producto bruto total mostró una baja importante. 
En suma, hemos visto, primero, que la participación del sector de servicios aumentó notablemente en el período 1986-1996, revirtiendo el marcado descenso que había registrado en el período previo. Segundo, la mayor parte de estos buenos resultados se debió a la intermediación directa. Tercero, en administración pública y educación/salud las tasas de crecimiento fueron muy insuficientes y la participación en la producción bruta se redujo de manera apreciable, lo que podría ser inquietante si se piensa que estas dos actividades son la base de la eficiencia de un país, esto es, de la coordinación y la productividad de la economía y de la sociedad.

Medida en función del PIB, entre 1986 y 1996 se elevó aún más la participación del sector de servicios, en alrededor de otros tres puntos porcentuales. El comercio se expandió en forma significativa, recuperándose de la caída que había experimentado en el período previo, y otro tanto sucedió con el transporte y en especial con las comunicaciones. En parte esto se originó en el fuerte ingreso de empresas de telecomunicaciones (Moguillanski, 1999). El financiamiento se mantuvo en los niveles relativamente altos alcanzados en 1986, con tasas de crecimiento medio similares a la del PIB. Es posible que en ese resultado hayan influido la privatización de los fondos de pensiones y la proliferación de estos fondos, pero también se vio favorecido por una importante afluencia de capitales de corto plazo (Uthoff, 2001). Medido en función del PIB, el crecimiento de la administración pública y la educación/salud se situó sistemáticamente a la zaga del crecimiento medio de la economía, no solo entre 1986 y 1996, sino también en el 2000 (Banco Central de Chile, varios años).

Pese a las satisfactorias tasas de crecimiento del período analizado, al parecer la economía todavía no privilegiaba los sectores tecnológicamente más complejos. Tanto la industria liviana como la pesada crecieron mucho menos que el promedio de la economía, perdiendo terreno frente a los servicios. Entre las industrias de mayor complejidad tecnológica, según la clasificación de Wyckoff y Sakurai (1992), al parecer solo aumentaron su participación en la producción las industrias de metales y no metales; en cambio, las de caucho, maquinaria, equipo de transporte y otras manufacturas parecen haber quedado a la zaga. Con todo, el financiamiento creció a una tasa bastante superior al promedio, lo que usualmente se considera un complemento necesario del progreso tecnológico. ${ }^{12}$

\footnotetext{
12 Véase también Albala-Bertrand (1999a).
}

Para concluir, pese a la caída de la participación del sector manufacturero y a la baja relativa del aporte efectuado por el incremento de las exportaciones, el acontecimiento más interesante del período parece haber sido el aumento generalizado de la intermediación directa en la economía en su conjunto. Esto quiere decir que actualmente las actividades económicas estarían más integradas que nunca antes; pero también podría significar que ahora la economía es más vulnerable a crisis parciales. En otras palabras, es posible que un shock recesivo en un sector determinado tenga efectos adversos mayores que en el pasado en el resto de la economía. Sin embargo, convendría evaluar si este aumento de la interdependencia directa puede atribuirse al juego de las actividades productivas en el interior del país o al ingreso de insumos importados. Por esta razón, en los párrafos que siguen consideraremos el tema de la interdependencia industrial general en el período 1986-1996 y examinaremos los eslabonamientos hacia atrás y hacia delante, tanto directos como indirectos.

\section{Los cambios estructurales en la interdepen- dencia industrial}

El nivel y la calidad de la interdependencia o eslabonamiento de las industrias guardan relación con el desarrollo. El mayor uso de insumos eficientes está asociado a la creciente complejidad del sistema económico, es decir, a que en la producción haya más ciclos de intermediación o interdependencia industrial, como sucede en los países desarrollados. Los eslabonamientos y la producción final también están positivamente correlacionados (cuadro 2). Al parecer, el aumento de los eslabonamientos intermedios en algunas industrias antecede el incremento de su producción final, especialmente cuando se trata de exportaciones de bienes manufacturados y de manufacturas pesadas o más complejas (Chenery y Syrquin, 1986).

El cuadro 2 muestra la variación (o tasas de crecimiento) de los eslabonamientos hacia atrás $(\triangle B L / B L)$ y hacia adelante $(\triangle F L / F L)$ en el período 1986-1996, distinguiendo entre contribuciones internas (DOM) e importadas (IMP), ambas a precios básicos constantes de 1986.

\section{a) Los eslabonamientos hacia atrás (BL)}

A manera de ilustración diremos que en el cuadro 2, bajo el encabezado "BL86" en la sección izquierda, la cifra 2,1 correspondiente al cobre indica que para producir una unidad de cobre (con valor constante equivalente a un peso) destinada a satisfacer demanda 
Chile: Tasas de variación 1986-1996 de los eslabonamientos hacia atrás y hacia adelante, por actividades productivas y por sectores ${ }^{a}$

(A precios básicos constantes en pesos de 1986)

\begin{tabular}{|c|c|c|c|c|c|c|c|c|c|c|c|c|c|c|c|}
\hline & & & & Eslabo & hamient & tos hacia & atrás & & & Eslal & onam & entos $h$ & acia adel & lante & \\
\hline Act. & productivas y sectores & $\Delta \mathrm{BL} / \mathrm{BL}(86)$ & DOM & IMP & BL86 & NBL96 & CV96 & SFD86 & $\Delta \mathrm{FL} / \mathrm{FL}(86)$ & DOM & IMP & FL86 & NFL96 & CV96 & SV86 \\
\hline 1 & Ind. primarias no mineras & 23 & 13 & 9 & 1,9 & 0,95 & 2,5 & 5 & 15 & 6 & 9 & 2,0 & 0,52 & 3,0 & 8 \\
\hline 2 & Cobre & 3 & -3 & 6 & 2,1 & 0,88 & 2,7 & 9 & -1 & -3 & 2 & 1,2 & 0,27 & 4,9 & 5 \\
\hline 3 & Combustibles & 90 & 32 & 58 & 2,1 & 1,62 & 2,2 & 0 & 545 & -89 & 633 & 7,7 & 11,19 & 1,4 & 1 \\
\hline 4 & Otros minerales & 5 & 0 & 5 & 1,4 & 0,57 & 3,6 & 5 & 2 & -3 & 5 & 1,4 & 0,33 & 3,5 & 5 \\
\hline 5 & Alimentos & 40 & 24 & 16 & 2,6 & 1,47 & 2,1 & 13 & 28 & 19 & 9 & 1,4 & 0,41 & 3,9 & 5 \\
\hline 6 & Textiles & 97 & 36 & 61 & 2,4 & 1,86 & 2,2 & 1 & 134 & 52 & 83 & 2,4 & 1,27 & 1,9 & 2 \\
\hline 7 & Vestuario & -4 & -15 & 11 & 2,6 & 0,99 & 2,3 & 2 & 80 & 46 & 34 & 1,2 & 0,47 & 2,7 & 1 \\
\hline 8 & Leña/madera & 48 & 37 & 11 & 2,1 & 1,23 & 2,0 & 2 & 16 & 4 & 12 & 2,1 & 0,54 & 2,5 & 2 \\
\hline 9 & Papel/imprenta & -2 & -7 & 5 & 2,1 & 0,83 & 2,5 & 1 & 44 & 20 & 24 & 2,2 & 0,69 & 1,8 & 1 \\
\hline 10 & Cuero & -40 & -37 & -3 & 1,9 & 0,45 & 4,7 & 0 & 33 & 16 & 17 & 1,7 & 0,52 & 2,2 & 0 \\
\hline 11 & Caucho & 41 & 18 & 23 & 1,8 & 1,02 & 2,4 & 1 & 82 & 23 & 58 & 2,5 & 1,04 & 1,6 & 1 \\
\hline 12 & Productos químicos & 8 & 1 & 6 & 2,2 & 0,95 & 2,9 & 2 & 30 & 6 & 25 & 4,1 & 1,20 & 1,4 & 3 \\
\hline 13 & Refinación de petróleo & 61 & -1 & 62 & 2,9 & 1,87 & 2,0 & 1 & 30 & -19 & 49 & 3,3 & 0,95 & 1,7 & 3 \\
\hline 15 & Metales & 0 & -11 & 11 & 2,1 & 0,83 & 3,1 & 2 & 8 & -25 & 33 & 3,1 & 0,76 & 2,1 & 3 \\
\hline 16 & Maquinaria & 24 & 6 & 18 & 1,4 & 0,72 & 3,3 & 2 & 79 & 7 & 72 & 2,7 & 1,09 & 1,4 & 3 \\
\hline 17 & Equipos de transporte & 30 & 8 & 22 & 1,6 & 0,82 & 3,2 & 1 & 60 & 7 & 53 & 2,6 & 0,92 & 1,8 & 2 \\
\hline 18 & Otras manufacturas & 19 & 3 & 16 & 2,2 & 1,04 & 2,1 & 1 & 55 & 33 & 22 & 2,0 & 0,71 & 1,7 & 1 \\
\hline 19 & Construcción & 31 & 16 & 15 & 2,1 & 1,08 & 1,9 & 8 & -3 & -12 & 9 & 1,4 & 0,30 & 3,9 & 5 \\
\hline 20 & Servicios de utilidad pública & 37 & 13 & 24 & 1,9 & 1,06 & 3,0 & 1 & 19 & -9 & 28 & 2,6 & 0,69 & 2,6 & 2 \\
\hline 21 & Comercio/restaurantes/hoteles & 19 & 10 & 9 & 1,8 & 0,83 & 2,7 & 13 & -7 & -20 & 13 & 1,8 & 0,37 & 3,4 & 13 \\
\hline 22 & Finanzas & 23 & 17 & 6 & 1,9 & 0,93 & 2,7 & 1 & -12 & -32 & 20 & 2,3 & 0,45 & 3,1 & 1 \\
\hline 23 & Serv. a empr./bienes raíces & 15 & 9 & 6 & 1,4 & 0,64 & 3,5 & 7 & 31 & 1 & 30 & 2,0 & 0,59 & 2,1 & 10 \\
\hline 24 & Transportes & 17 & 4 & 13 & 2,2 & 1,02 & 2,5 & 6 & 14 & -23 & 38 & 2,2 & 0,57 & 2,6 & 7 \\
\hline 25 & Comunicaciones & 16 & 17 & 0 & 1,4 & 0,67 & 3,6 & 1 & -11 & -27 & 15 & 2,2 & 0,44 & 3,1 & 1 \\
\hline 26 & Administración pública & 44 & 31 & 14 & 1,8 & 1,01 & 2,0 & 7 & 1 & 1 & 0 & 1,0 & 0,23 & 5,1 & 5 \\
\hline 27 & Educación/salud & 34 & 25 & 9 & 1,4 & 0,77 & 2,7 & 7 & 4 & 2 & 1 & 1,0 & 0,24 & 4,9 & 6 \\
\hline 28 & Otros servicios & 23 & 14 & 9 & 1,7 & 0,85 & 2,5 & 2 & 77 & 70 & 7 & 1,3 & 0,50 & 2,4 & 2 \\
\hline II. & Manufactura:Liviana (5-10) & 36 & 19 & 17 & 2,5 & 1,14 & 2,4 & 20 & 46 & 23 & 23 & 1,8 & 0,65 & 3,0 & 12 \\
\hline & Pesada (11-18) & 23 & 2 & 21 & 2,0 & 1,04 & 2,9 & 10 & 41 & -3 & 43 & 3,1 & 0,92 & 1,7 & 16 \\
\hline & Construcción (19) ${ }^{\mathrm{b}}$ & 31 & 16 & 15 & 2,1 & 1,08 & 1,9 & 8 & -3 & -12 & 9 & 1,4 & 0,30 & 3,9 & 5 \\
\hline III. & Sector servicios (20-28) & 25 & 15 & 10 & 1,7 & 0,87 & 2,8 & 44 & 10 & -8 & 18 & 1,8 & 0,45 & 3,2 & 47 \\
\hline & Promedio $^{c}$ & 25 & 12 & 12 & 2,0 & 1,00 & 2,6 & 100 & 25 & -3 & 28 & 2,0 & 1,00 & 2,9 & 100 \\
\hline
\end{tabular}

Fuente: Elaboración propia con datos del Banco Central de Chile (1993 y 2001a).

a BP : Precios básicos.

$\Delta \quad$ : Variación.

DOM : Internos (puntos porcentuales).

IMP : Importados (puntos porcentuales).

BL : Eslabonamientos hacia atrás (pesos).

NBL : Eslabonamientos hacia atrás normalizados (pesos).

CV96 : Coeficiente de variación en 1996 (pesos).

SFD : Participación en la demanda interna final (porcentaje).

FL : Eslabonamientos hacia adelante (pesos).

NFL : Eslabonamientos hacia adelante normalizados (pesos).

CV96 : Coeficiente de variación en 1996 (pesos).

SV : Participación en el valor agregado (porcentaje).

b La construcción está incluida en el sector manufacturero.

c Suma de SFD86 y SV86. 
final se necesitan 2,1 unidades de insumos intermedios directos e indirectos. El valor normalizado de $B L$ para el mismo año y la misma industria, bajo el encabezado "NBL86", muestra una cifra inferior a uno, lo que significa que los eslabonamientos hacia atrás de la industria del cobre son inferiores al promedio de la economía; esto no es de extrañar, puesto que en Chile dicha industria constituye un "enclave de demanda", es decir, genera escasa demanda de insumos producidos por otras industrias. Dicho valor muestra también una homogeneidad media, puesto que su coeficiente de variación se aproxima al promedio. En 1996, las ramas industriales que destacaban por su liderazgo o empuje eran la alimentaria y la textil; también la de combustibles y de refinación de petróleo, pero estas últimas partieron de una base muy baja, ya que en 1986 su participación en el PIB era escasa o insignificante. Las ramas más rezagadas eran otros minerales, cuero, maquinaria, servicios a empresas/bienes raíces, comunicaciones y educación/salud. La importancia de los insumos importados para la mayor parte del grupo de industrias que lideraba, en especial la refinación de petróleo, y el papel clave de esos insumos en la mayoría de las demás industrias, podrían tener efectos adversos en la integración productiva del país.

Cabe señalar que en 1986-1996, siguiendo una tendencia similar a la del período anterior, los eslabonamientos hacia atrás parecen haber aumentado en promedio otro $25 \%$. Esto quiere decir que cuando la economía produjo internamente 100 unidades de productos finales, generó en promedio casi 25 unidades más de demanda intermedia hacia atrás en 1996 que en 1986. Esto se debió a que aumentaron en especial los eslabonamientos hacia atrás de la construcción y la industria liviana. Sin embargo, buena parte del aumento provino de la intermediación importada, sobre todo en la industria pesada. En general, los bienes intermedios importados y nacionales parecieron compartir equitativamente la generación del incremento. No obstante, en especial en la industria pesada, los insumos más importantes son importados y no nacionales. Esta tendencia, que ya se había manifestado en el período anterior, pareció ser menos marcada en el período que estamos analizando. Esto podría influir tanto en la vulnerabilidad externa como en el desarrollo tecnológico endógeno (Albala-Bertrand, 1999b).

A nivel desagregado se observa, primero, que en el sector primario el aumento de los eslabonamientos hacia atrás se dio principalmente en los combustibles y en segundo lugar en las industrias no mineras. El incremento en los combustibles fue significativo de- bido tanto a la intermediación interna como a la importada, pero generalmente fue esta última la que llevó el mayor peso. Contrariamente a lo sucedido en el período anterior, en 1986-1996 el cobre mostró un pequeño aumento de $3 \%$, que resultó de una caída de tres puntos porcentuales en la intermediación interna y de un alza de $6 \%$ en la intermediación importada.

En segundo lugar, los eslabonamientos hacia atrás de la industria liviana, que en 1977-1986 se redujeron significativamente, acusaron un incremento apreciable en 1986-1996, en especial gracias a la industria alimentaria y a la textil. Sin embargo, las industrias de prendas de vestir, papel/imprenta y cuero registraron caídas originadas totalmente en la significativa baja de la intermediación interna.

En tercer lugar, la industria pesada exhibió en el período estudiado un importante incremento medio de los eslabonamiento hacia atrás, pero debido casi íntegramente a la intermediación importada. Los efectos positivos más marcados se originaron en las industrias de caucho, refinación de petróleo, equipos de transporte y maquinaria, con una contribución menor de las fuentes internas. Por su parte, las industrias de metales registraron un crecimiento cero debido a que el reemplazo de bienes nacionales por insumos importados fue de 11 puntos porcentuales. En la construcción, la intermediación fue más equilibrada que en los demás sectores.

Finalmente, en el sector terciario se observó un aumento de los eslabonamientos hacia atrás, siendo el aporte de las fuentes externas mayor que el de las internas, excepto en los transportes.

Lo esencial es que si bien la intermediación importada representó el 50\% del cambio total, en el segmento tecnológicamente más avanzado de la economía la cifra se elevó casi al $90 \%$. Esta penetración de insumos importados parece estar afectando con fuerza a todos los componentes de la industria pesada. Pese a que esto puede atentar contra la sostenibilidad endógena, talvez contribuya a mantener la competitividad de las industrias que utilizan insumos importados de mayor complejidad tecnológica. Al parecer, la economía abierta chilena va hacia una mayor dependencia de insumos importados para atender a las necesidades normales de producción. En el período 19771986 se observó una caída de la intermediación hacia atrás interna, que a la sazón se consideró una desintermediación generalizada (Albala-Bertrand, 1999b). Esta situación parece haberse revertido en la mayoría de las actividades industriales, salvo en la industria pesada. 
Más allá de su contribución al empleo, el aumento de los eslabonamientos hacia atrás importados solo benefician directamente al exportador extranjero y al importador local, y poco o nada favorecen la interdependencia productiva endógena de las industrias. Desde este punto de vista, los posibles efectos beneficiosos de un incremento de la demanda de insumos se filtran fuera de la economía a los exportadores extranjeros y al comercio con los importadores locales, pero no ayudan mucho a la producción manufacturera interna. Esto podría ser inadecuado para propiciar el desarrollo manufacturero autóctono. ${ }^{13}$

\section{b) Los eslabonamientos hacia adelante (FL)}

De manera similar a lo que sucede con los eslabonamientos hacia atrás, de una economía con una base industrial creciente y dinámica se espera una amplia y vigorosa oferta interna de bienes intermedios locales. Naturalmente, esto no impide que las empresas eleven la producción destinada a satisfacer la demanda final y a exportar, siempre que, a igualdad de condiciones, no lo hagan de manera generalizada e intensa a expensas de la oferta interna de insumos (Albala-Bertrand, 1999b). En el período analizado los eslabonamientos hacia adelante aumentaron al mismo ritmo que aquellos hacia atrás, solo que todo su incremento se produjo a costa de la oferta interna de insumos importados, quitando además tres puntos porcentuales a los insumos de origen interno. Si se excluye el par de industrias que exhibieron la variación más alta en los eslabonamientos hacia adelante (la de combustibles y la textil), el incremento resulta moderado pero mantiene el mismo patrón. Al parecer hubo una caída generalizada de los eslabonamientos hacia adelante de origen interno. Esto quiere decir que el aumento de la oferta de insumos provino de bienes importados, en desmedro de la oferta interna. En otras palabras, los aumentos de valor agregado han creado oportunidades para los proveedores de insumos importados y no para los productores locales.

Esto puede significar que, en la práctica, muchos productores locales hayan renunciado a la posibilidad de abastecer el mercado interno con producción nacional y hayan optado por la comercialización de importaciones. Esta tendencia ya se había observado en

\footnotetext{
${ }^{13} \mathrm{Al}$ igual que en el caso extremo de una economía manufacturera con producción en serie que se dedica exclusivamente a ensamblar partes fabricadas en el extranjero, pero compra esas partes a importadores locales que pertenecen a otros sectores.
}

1977-1986, años en los cuales la intermediación de fuentes internas fue negativa en casi todas las industrias consideradas. En el período 1986-1996 dicha tendencia no había desaparecido del todo. En un buen número de industrias la variación de la intermediación interna fue negativa, mientras que el cambio en la oferta de insumos importados fue positivo. Para todas las industrias, salvo las livianas, la contribución de la intermediación interna fue negativa. Las ramas más afectadas fueron las de combustibles, metales, financiamiento, transporte y comunicaciones. Esto indica a la vez que se consolidó la falta de intermediación interna observada en el período anterior y que hubo un distanciamiento aun mayor del tipo de proceso de integración interna vivido por las economías taiwanesa y sudcoreana (Albala-Bertrand, 1999b). Como en el caso de los eslabonamientos hacia atrás, esto refleja una generalizada desintermediación interna.

Como conclusión general respecto de los eslabonamientos hacia atrás y hacia adelante, puede decirse que la marcada penetración de importaciones puede tener consecuencias dispares en el dinamismo de una economía en crecimiento. Por un lado, la economía puede escoger las fuentes de insumos externos más eficientes y utilizar insumos tecnológicos más complejos, lo que puede ser provechoso. Por otro lado, es posible que la economía se torne más vulnerable a las fluctuaciones externas, lo que puede llevar a mayor inestabilidad (Damill, Fanelli y Frenkel, 1996; Chang y Grabel, 2004). Y no menos importante, también es posible que la economía pierda bastante eficiencia al no explotar la sincronización interna de los insumos necesarios y sus especificaciones, sofocando así el potencial autóctono de desarrollo tecnológico. La decisión de evitar esta anomalía, que guarda relación con políticas de insumos deliberadas, contribuyó decisivamente a una coordinación eficaz de las necesidades de las industrias con la oferta de los proveedores internos en la economía taiwanesa y la sudcoreana. También ayudó a elevar el nivel de complejidad de su progreso tecnológico autóctono. ${ }^{14}$ En Chile, este aspecto parece haberse debilitado aún más en el período 1986-1996 y en general hacia el 2000 (Banco Central de Chile, varios años), lo que podría hacer más difícil sustentar un crecimiento tecnológicamente avanzado. En otras palabras, para que la economía chilena pueda aprovechar

\footnotetext{
14 Véase Kubo, de Melo y otros (1986); Wyckloff y Sakurai (1992); Chang (1996); Stiglitz (1996); Albala-Bertrand (1999b); Islam y Choudhury (2000); Chang y Grabel (2004).
} 
plenamente su potencial de eficiencia y desarrollo tecnológico a la manera de la economía taiwanesa y la República de Corea, tiene que equilibrar el acceso a diversas fuentes de insumos externos con el apoyo a fuentes de insumos internos. Dado que tanto las estrategias de liberalización como las estrategias reguladoras

\section{IV}

\section{Conclusiones}

Hasta 1986, Chile parecía haberse apartado de la clase de estructura productiva exitosa exhibida por la economía taiwanesa y la República de Corea en los 15 a 20 años que siguieron al inicio de su desarrollo acelerado o, por lo menos, parecía no haber avanzado hacia ella (Albala-Bertrand, 1999a y 1999b). Casi veinte años después de iniciada una amplia liberalización económica, la estructura productiva de Chile no parece haberse equiparado con las de esas economías del sudeste asiático. De hecho, parece haberse alejado aun más de ellas, especialmente en lo que se refiere a la complejidad tecnológica endógena. A largo plazo, esto puede afectar el progreso tecnológico, la sostenibilidad del desarrollo y la estabilidad de la economía en general.

Según cifras de la CEPAL, en 1996 la participación de la industria manufacturera en el PIB se aproximó al $20 \%$ y la de la industria pesada se situó en torno al $2 \%$. A su vez, la participación de las exportaciones en el PIB se elevó a cerca de $25 \%$, pero solo un $3 \%$ de esas exportaciones correspondió a manufacturas. Entretanto, la participación de la industria manufacturera pesada en el PIB continuó siendo insignificante. Hacia el 2000 este patrón no había variado y hasta la fecha no parece haberse modificado significativamente (CEPAL, tienen costos y beneficios, las autoridades encargadas de formular las políticas deben mantener un delicado equilibrio entre unas y otras para asegurar, o al menos no frustrar, la sostenibilidad endógena de una mayor complejidad tecnológica interna y la eficiencia vinculada a la coordinación interindustrial. varios años). Dado que la economía chilena perdió dinamismo o entró en un ciclo recesivo después de 1996, con una tasa media de crecimiento cercana a la mitad de la alcanzada en el período anterior, es poco probable que se haya acrecentado la intermediación interna o que hayan arraigado industrias manufactureras tecnológicamente complejas. Por lo demás, las insuficiencias señaladas podrían tener efectos adversos en la distribución del ingreso, el combate contra la pobreza y el bienestar general, o bien demorar los avances en estas áreas. ${ }^{15}$

Es por ello que, pese a exhibir otros logros, el modelo de desarrollo económico adoptado por Chile aún no parece favorecer la interdependencia endógena, especialmente aquella encabezada por industrias tecnológicamente complejas. Tampoco parece estar orientado hacia el tipo de exportaciones capaces de sustentar y estimular un desarrollo industrial dinámico orientado a los mercados externos. En otras palabras, la economía chilena aún parece ser relativamente débil como para sustentar un desarrollo económico y tecnológico de mayor complejidad.

(Traducido del inglés)
${ }^{15}$ Las cifras sobre los niveles de pobreza y la distribución del ingreso en la economía taiwanesa y en la República de Corea se cuentan entre las mejores del mundo (Islam y Choudhury, 2000; Sundrum, 1990). En Chile, tras casi dos décadas de cambios estructurales, la pobreza y la desigualdad siguieron alcanzando niveles sin precedentes (Albala-Bertrand, 1996). Aunque la pobreza se ha reducido tras el retorno a la democracia en 1990, la distribución del ingreso ha seguido deteriorándose o se han mantenido las desigualdades heredadas de la dictadura. Tanto es así que Chile ocupa el noveno lugar en el mundo entre los países con peor distribución del ingreso (PNUD, 2004; MIDEPLAN, 2000; Pizarro, Raczynski y Vial, 1996). 
Achurra, M. (1995): La experiencia de un nuevo producto de exportación: los salmones, en P. Meller y R. Sáez (comps.), Auge exportador chileno: lecciones y desafíos futuros, Santiago de Chile, Dolmen Ediciones.

Agosin, M. (1997): Export Performance in Chile: Lessons for Africa, Wider Working Papers, $N^{\circ} 144$, Helsinki, Instituto Mundial de Investigaciones de Economía del Desarrollo.

Albala-Bertrand, J.M. (1996): Structural Change in Chile: 1960-1990. An Input-output Approach, Working Paper Series, No 354, Londres, Universidad de Londres.

(1999a): Structural change in Chile: 1960-1990, Economic Systems Research, vol. 11, No 3, Londres, Routledge.

(1999b): Industrial interdependence change in Chile: 19601990. A comparison with Taiwan and South Korea, International Review of Applied Economics, vol. 13, No 2, Londres, Routledge.

Banco Central de Chile (1993): Matriz de insumo-producto para la economía chilena 1986, Santiago de Chile.

(2001a): Matriz de insumo-producto para la economía chilena 1996, Santiago de Chile.

(2001b): Cuentas nacionales, 1999, Santiago de Chile. (varios años): Boletín mensual, Santiago de Chile.

Bulmer-Thomas, V. (1982): Input-Output Analysis in Developing Countries, Nueva York, John Wiley.

CEPAL (Comisión Económica para América Latina y el Caribe) (varios años): Anuario estadístico de América Latina y el Caribe, Santiago de Chile.

Ciaschini, M. (comp.) (1988): Input-Output Analysis: Current Developments, Londres, Chapman and Hall.

Chang, H.J. (1996): The Political Economy of Industrial Policy, Londres, Macmillan.

Chang, H.J. e I. Grabel (2004): Reclaiming Development, Londres, Zed Books.

Chenery, H. (1960): Patterns of industrial growth, American Economic Review, vol. 50, No 4, Nashville, Tennessee, American Economic Association.

Chenery, H. y M. Syrquin (1986): Typical patterns of transformation, en H. Chenery, S. Robinson y M. Syrquin (comps.), Industrialization and Growth, Nueva York, Oxford University Press.

Damill, M., J.M. Fanelli y R. Frenkel (1996): De México a México: el desempeño de América Latina en los noventa, Desarrollo económico, vol. 36, número especial, Buenos Aires, Instituto de Desarrollo Económico y Social (IDES).

Dervis, K., J. de Melo y S. Robinson (1982): General Equilibrium Models for Development Policy, Nueva York, Cambridge University Press.

Dietzenbacher, E. y B. Los (1998): Structural decomposition techniques: sense and sensitivity, Economic Systems Research, vol. 10, $\mathrm{N}^{\mathrm{o}} 4$, Londres, Routledge.

Ffrench-Davis, R. y B. Stalling (comps.) (2001): Reformas, crecimiento y políticas sociales en Chile desde 1973, Santiago de Chile, CEPAL/LOM Ediciones.

Ffrench-Davis, R. y R. Sáez (1995): Comercio y desarrollo industrial en Chile, Colección Estudios de CIEPLAN, No 41, Santiago de Chile, diciembre.

Hirschman, A. (1958): The Strategy of Economic Development, New Haven, Yale University Press.

(1977): Enfoque generalizado del desarrollo por medio de enlaces, con referencia general a los productos básicos, $\mathrm{El}$ trimestre económico, vol. 44(1), No 173, México, D.F., Fondo de Cultura Económica, enero-marzo.

Islam, I. y A. Choudhury (2000): The political Economy of East Asia. Post-Crisis Debates, Oxford, Oxford University Press.
Krueger, A. (1990): Government failures in development, Journal of Economic Perspectives, vol. 4, No 3, Nashville, Tennessee, American Economic Association.

Kubo, Y., J. de Melo y otros (1986): Interdependence and industrial structure, en H. Chenery, S. Robinson y M. Syrquin (comps.), Industrialization and Growth, Nueva York, Oxford University Press.

Lall, S. (1996): Learning from the Asian Tigers: Studies in Technological and Industrial Policy, Londres, Macmillan Press. (1997): Selective policies for export promotion: lesson from Asian Tigers, Research for Action, $\mathrm{N}^{\circ} 43$, Helsinki, Instituto Mundial de Investigaciones de Economía del Desarrollo.

MIDEPLAN (Ministerio de Planificación y Cooperación) (2000): Encuesta CASEN 2000, Santiago de Chile.

Moguillansky, G. (1999): La inversión en Chile: ¿el fin de un ciclo en expansión?, Santiago de Chile, CEPAL/Fondo de Cultura Económica.

Mujica, J. (1989): Deindustrialization in Chile, Boulder, Colorado, Westview.

Ominami, C. (1991): Deindustrialization and industrial restructuring in Latin America: the examples of Argentina, Brazil and Chile, en P. Meller (comp.), The Latin American Development Debate: Neostructuralism, Neomonetarism and Adjustment Processes, Boulder, Colorado, Westview.

Pizarro, C., D. Raczynski y J. Vial (comps.) (1996): Social and Economic Policies in Chile's Transition to Democracy, Santiago de Chile, Corporación de Investigaciones Económicas para Latinoamérica (CIEPLAN).

PNUD (Programa de las Naciones Unidas para el Desarrollo) (2004): Informe sobre desarrollo humano 2004, Nueva York.

Sakurai, N. (1990): Methodology of Multisectoral Growth Decomposition Models: A Survey and Extensions, OECD Discussion Paper, № 1, París, Organización de Cooperación y Desarrollo Económicos (OCDE).

Stiglitz, J. (1996): Some lessons from the East Asian miracle, The World Bank Research Observer, vol. 11, № 2, Washington, D.C., Banco Mundial, agosto.

(1998): More Instruments and Broader Goals: Moving toward the Post-Washington Consensus, Helsinki, Universidad de las Naciones Unidas/ Instituto Mundial de Investigaciones de Economía del Desarrollo.

Stiglitz, J. y M. Uy (1996): Financial markets, public policy and the East Asian miracle, The World Bank Research Observer, vol. 11, No 2, Washington, D.C., Banco Mundial.

Sundrum, R.M. (1990): Income Distribution on Less Developed Countries, Londres, Routledge.

Syrquin, M. (1992): Linkages and the strategy of development, en S. Teitel (comp.), Towards a New Development Strategy for Latin America, Washington, D.C, Banco Interamericano de Desarrollo (BID).

Uthoff, A. (2001): La reforma del sistema de pensiones y su impacto en el mercado de capitales, en R. Ffrench-Davis y B. Stallings (comps.), Reformas, crecimiento y políticas sociales en Chile desde 1973, Santiago de Chile, CEPAL/LOM Ediciones.

Wade, R. (1990): Governing the Market: Economic Theory and the Role of the Government in East Asian Industrialization, Princeton, Princeton University Press.

Williamson, J. (comp.) (1990): Latin American Adjustment. How Much Has Happened?, Washington, D.C., Instituto de Economía Internacional.

Wyckoff, A. y N. Sakurai (1992): Structural Change and Industrial Performance, París, Organización de Cooperación y Desarrollo Económicos (OCDE). 